\title{
Augmented Reality Facilitating Visual Literacy for Engagement with Science in Museums
}

\author{
Diana Marques \\ University of Porto \\ Faculty of Engineering \\ Rua Dr. Roberto Frias, s/n \\ 4200-465 Porto, Portugal \\ diana@dianamarques.com
}

\author{
Robert Costello \\ National Museum of Natural History \\ Smithsonian Institution \\ 10th St. \& Constitution Ave. NW \\ Washington, D.C. 20560, USA \\ costellr@si.edu
}

\author{
José Azevedo \\ University of Porto \\ Faculty of Letters \\ Via Panorâmica, s/n \\ 4150-564 Porto, Portugal \\ azevedo@letras.up.pt
}

\begin{abstract}
Visual literacy has not been a common topic in natural history museums. The visitor experience in those institutions is nonetheless object centred and a more visually connection could lead to stronger emotional experiences that promote lasting memories and the construction of meanings. Visual communication has also a universal character, which is of relevance in international environments like museums, where visitors present an array of backgrounds, motivations and disabilities. Augmented Reality, a promising technology in the rise of visitors' engagement, can offer strong visual interpretive experiences and is being used for the renovation of a Victorian-age skeleton exhibit at the Smithsonian's National Museum of Natural History. The Osteology Hall augmented reality app will perform with real time tracking and object recognition to enable the visualization of the animals' unique anatomical features and the roles they play in the environment, to motivate learning, and increase the enjoyment and memorableness of the experience.
\end{abstract}

Augmented reality. Visual literacy. Natural History Museums. Osteology exhibits. Scientific animation. Mobile technology.

\section{INTRODUCTION}

Visual literacy has been an important topic in the core curricula of art education since it is generally understood as the ability to construct meaning from images, by truly looking and interpreting them. Students are expected to interpret visual representations beyond the immediate sensorial experience through the development of visual critical thinking skills (Perkins 1994). Visual literacy also plays out in the public spaces of art museums where educators strive to raise visitor appreciation and understanding of the original artworks by facilitating their observation and interpretation skills, with an intention to have these skills and museum experiences transfer into their daily lives (Rice 1988).

In the realm of science education, visual literacy is also emphasized for communicating ideas and information, especially since the prominence in the curricula has shifted to the processes of science and since increases in the computational power of computers have made the creation of dynamic models and simulations possible (Gilbert 2005). However, in the learning of science in informal environments, such as natural history museums, visual literacy isn't such a common affair. As with art museums, natural history exhibits revolve around objects; therefore, a similar aesthetic influences the visitor experience and there are also parallels in the way museum specialists in both types of museums facilitate visitors' visual interpretations with ancillary materials (Bain \& Ellenbogen 2002, Jones 2002). But the presence of support material in the form of explanatory texts, graphic panels, audio and video, is far superior when museum professionals interact with visitors. The engagement of museum audiences with science exhibits has traditionally followed structured narratives and the simplification of messages, through an abundance of diverse visual and non-visual forms of communication.

\section{VISUAL LITERACY IN THE MUSEUM}

A case can be made for a greater emphasis on visual literacy at natural history museums.

Objects in science exhibition displays possess a value on their own that doesn't rely exclusively on their context within a scientific narrative. A more visually based connection to objects, in less structured ways and with less supporting text, audio and video based interpretive material, could lead to enticing experiences. Learning goals have become the driver for many of the decisions that take place during exhibit conception; but greater 
attention paid to the visitor experience and to the benefits of stronger emotional experiences with lasting memories that derive from objects and images alone, could be, for the museum visitor, the preferred experience.

Additionally, a more significant reliance on images overcomes the obstacles of the written and spoken language, which presents challenges to visitors that either do not speak the language natively, or do not grasp specialized vocabulary. In contrast, there is a universal character to visual communication, which is of relevance in international environments like museums, where visitors present an array of backgrounds, motivations and disabilities. There is also the everlasting debate on the amount of text reading that actually takes place in the museum (Borun \& Miller 1980, McManus 1989), which is only aggravated when the subject is in a specialized field of science featuring its obscure terminology - when such vocabularies are used they can be regarded as a communication barrier and when simplified can be the target of critics for "dumbing down" science.

A suggested approach to the facilitation of visual literacy in natural history museums, similar to what is done in art institutions, has been to deliver programs by trained staff, emphasizing the role of collection specimens in current scientific research and how they can be observed and understood (Jones 2002). Nonetheless it is a solution with a limited reach and may not be affordable, which places visitor-centric exhibit design through visual communication as a more effective point of contact with the visitors.

The duration of a visit to an exhibit within a museum is typically brief and the experience is dependent on many factors like prior experience, knowledge, interest and social relationships (Falk \& Dierking 2000). Nevertheless, tapping into the existing visual literacy capacities of the visitors by offering visually impacting active experiences, may contribute to higher levels of engagement and relevant construction of meanings about science.

Technology is now integrated into museum exhibits unlike ever before and may be key in the facilitation of visual literacy. In particular mobile technology which is reinventing the museum's relationship with its audiences (Proctor 2012) - mobile devices are ubiquitous, a well known medium for visitors in general and capable of offering singular and relevant experiences (Kahr-Højland 2006). In fact, visual literacy has close ties to mobile technology since users respond to visuals with simple but effective gestures that relate action to understanding and interpretation of images.

Augmented Reality, an emerging technology in the museum interpretive toolkit, not only can be implemented through handheld devices but can also have a strong visual grounding, turning it into a powerful tool for the facilitation of visual literacy in museums.

\subsection{Augmented Reality}

Augmented Reality technology is one of the most recent promises in the rise of visitor's engagement. It was originally defined as a user experience where virtual objects are superimposed upon the real world, thus supplementing reality (Azuma 1997). And when used on a mobile technology platform, the displays act as a window or magnifying glass that show the surrounding environment with an augmented overlay.

It has even been considered a perfect medium for museum innovation (Schavemaker 2012), promising a technically advanced seamless interaction between visitors, collection objects and their data (Weng et al. 2011), putting interaction and unique personal experiences at the core (KahrHøjland 2006). Augmented reality can truly blend digital media with the physical space of the museum to a point where museum professionals can shape the involvement of the audiences with the objects on display (Parry 2012). The 2012 Museum Edition of the New Media Consortium Horizon report forecasts the technology to be broadly adopted in two-to-three years (Johnson et al. 2012), which concurs with the endorsements in current surveys of the museum community (Tallon 2013).

As mentioned, within museum exhibits, designers move visitors beyond the primarily aesthetic experience of viewing objects (the contact with the collection object) to a more passive and directed interpretive experience using secondary materials, for example graphic panels, explanatory text, videos and animations. But this supplemental material does not interact directly with the objects and the visitor must make the decision to invest further time and effort in turning away from the object to read the graphic panel, make sense around it, and then return to the object to construct meaning. Through augmented reality and especially with its use on handheld devices, the experiential and interpretive aspects can merge and generate a singularly integrated, visually interpretive museum experience (Snyder \& Elinich 2010).

With this spatial and temporal synchronization of reality and virtuality through imagery, the visitor native skills involved in understanding visuals and extracting meanings are particularly enabled. 


\section{THE OSTEOLOGY HALL}

At the Smithsonian's National Museum of Natural History (NMNH) the authors are conducting a research project, currently in its production stage, involving the creation of a mobile augmented reality experience.

The Osteology Hall, a Victorian-age permanent exhibit of the Museum, contains over 300 skeletons of mostly extant species from all modern vertebrate groups (mammals, birds, amphibians, reptiles and fishes). Some specimens were collected or acquired in the 19th century and featured at the Paleontology and Comparative Anatomy exhibit, 1882, at the US National Museum or displayed at world expositions. Some other specimens were later added when the exhibit was transferred and re-opened in 1911 at the newly built $\mathrm{NMNH}$ and rearranged in the 1960 s to its current configuration, i.e. mostly inside glass cases, in a sequence of five connecting rooms (Figure 1).

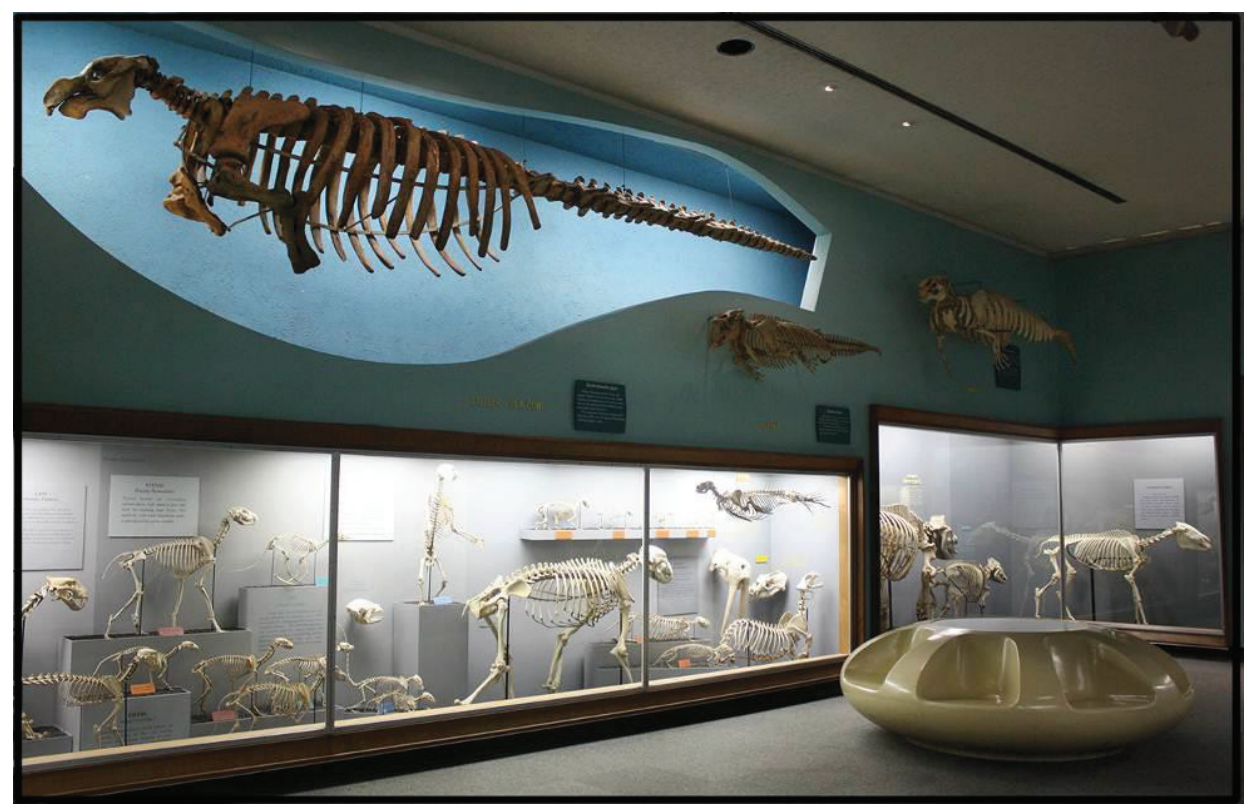

Figure 1: Mammal room at the Osteology Hall

Despite its biological and historical value, the exhibit no longer meets visitor expectations for interactivity and interpretation, it was conceived before the current frameworks were developed for engaging with audiences. The only information available is textual, containing over-specialized, outdated terms, and the exhibit does not promote reading as preliminary observations have informed. Further exacerbating visitor interpretation is the spatial separation of specimens from graphic panels and the scholarly construction of the texts is not in narrative form nor does it lend itself to promoting dialogue among visitors; in fact no question marks "?" can be found anywhere in the text on labels.

Research done at skeleton exhibits in natural history museums and zoos reveals that the majority of visitors are able to mentally skin the specimens and identify the skeletons with the name of the animals; but seldom do they know much about the skeletons per se or can they name its functions beyond the role of supporting the animal's body (Tunnicliffe 1998). Museum exhibits displaying skeletons are advised to have an orientation section that fleshes out details of the component parts of skeletons and highlights their functions (Tunnicliffe \& Scott 2003); it should be the museum focus to help visitors look at, think about, and interpret specimens, i.e. facilitating their visual literacy capacities (Jones 2002). In fact, one of the purposes of the digital makeover of the Osteology Hall is to integrate this kind of interpretation into the skeletal displays.

\subsection{Digital Renovation}

The renovation of the visitor experience entails the creation of a dedicated mobile app that can be used to interact with the exhibit through augmented reality. A variety of content - particularly still and moving images and binaural audio - is triggered at both the case and specimen levels and registered to them to enable the visualization of unique anatomical features and the roles they play in the environment.

For example, visitors approaching the Arboreal Birds display case will be able to see the content triggered by the Pileated Woodpecker skeleton, an 
overlaying animation of the external anatomy of the animal displaying its natural feeding behavior pecking to drill holes in the tree bark, listening to locate the insect larva and protruding its uniquely long tongue to capture them - and then a moving depiction of the skull registered to the specimen showing how the tongue is operated by one bone and several muscles that wrap around the skull and emerge through the lower part of the beak (Figure 2). This exclusive anatomical adaptation and feeding approach allow woodpeckers to exploit a largely untapped ecological resource of larval arthropods, and the app will convey this idea in an accurate and visually impacting way.

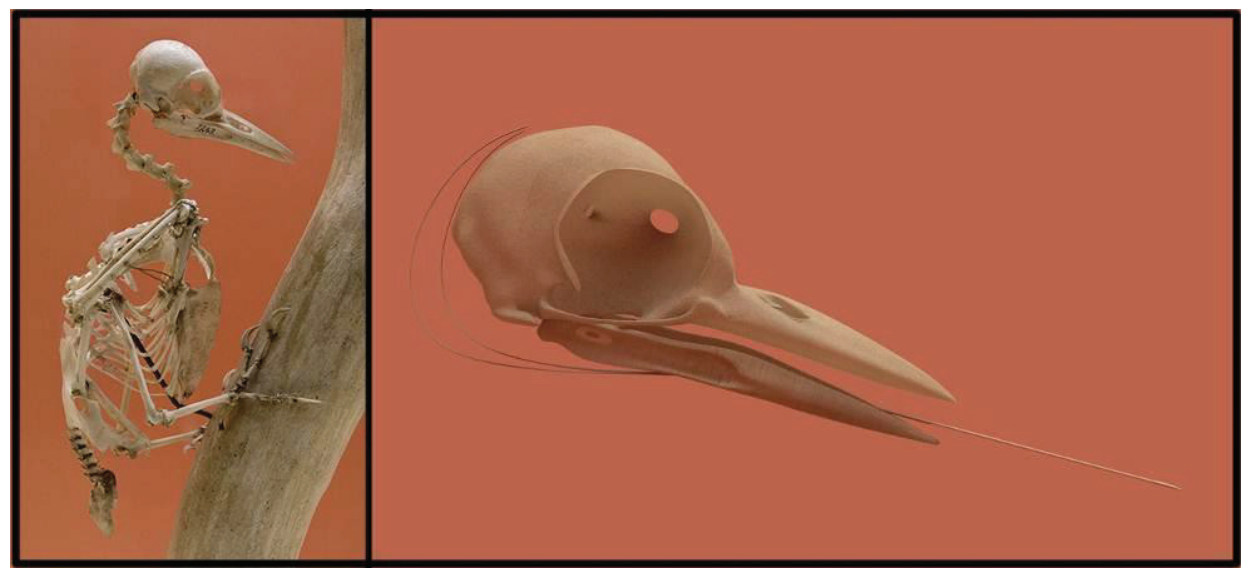

Figure 2: To the Pileated Woodpecker skeleton (left) will be registered a 3D computer animation of its skull (right, animation frame), to show the tongue mechanism

The ultimate goal of the augmented reality mobile app is to motivate learning, increase the enjoyment and memorableness of the experience, and to improve the communication of the main organizing principles of the exhibit - common descent, and functional and comparative anatomy - that support the biological concept of evolution. It is also being applied to an exhibit that was constructed decades ago and conceived more than a century past, deviating from the norm at museums where new digital technologies are usually integrated into new exhibitions.

Because there will be no physical renovation in the Osteology Hall, two quite distinct experiences will be available to the visitors: exploring the Victorianage Hall through a path that relies mostly on the objects and the aesthetic and sensorial connections they may foster; or downloading the app and walking through in an augmented fashion that better promotes the construction of meanings surrounding some skeletons.

\subsection{Facilitating Visual Literacy}

An ideal augmented reality experience achieves natural coexistence of virtuality and reality, enhancing the user's performance within and perception of the world. As such there is great requirement for geometrical consistency; care needs to be taken with the registration of the two visual stimuli that are carefully fused onto the same display environment. Human observers are very sensitive to alignment, so the accuracy of the overlaying imagery is critical (Azuma 1997).
The Osteology Hall app will perform with real time tracking and object recognition, meaning that the skeletons themselves are the trigger for the digital content and not a fiducial marker in the exhibit or the visitors' location. It is the most complex process of recognition involving the gathering of point cloud data, but it is also the method that better warrants an immersive experience, since regardless of the visitors' distance from and angle to the display cases, the digital content is always correctly registered and aligned.

Most of the processes involved in animal functional anatomy are complex and may be challenging to convey to a non-specialized audience. Linking the skeletons to the external appearance and behavior of the animals they belong to and even the natural contexts of those animals, is an approach that enables visitors to more easily recognize and relate to them. Studies have shown how dioramas in natural history museums can be more stimulating in the construction of narratives than singly displayed and taxidermied animals or their skeletons (Reiss \& Tunnicliffe 2011).

The digital content presented by the app will be mostly 3D computer graphics and animation that recreate a realistic look and motion of the subjects. Also, the choice of illustration and animation over real image video for most of the content is warranted by how difficult, time consuming and costly it can be to record directly from nature or even from animals in controlled environments. 


\subsection{Visitor Research}

The Osteology Hall app is under production and the collection of research data is underway with observation and tracking of the visitors to the exhibit in its current non-digitally enhanced state. Interviews are soon to begin for establishing baseline information about the audience's demographics, previous experience with augmented reality and for testing content preferences.

For research purposes the mobile app is being produced in two versions that feature the same content, only one version will deliver the content in a more traditional non-augmented way. Three variants of visitors will be surveyed pre or post visit: 1) those walking through the Hall without a device, 2) those walking through with tablet devices provided by the Museum with the non-augmented version of the app and 3) those who will carry the Museum's device and have the full augmented experience. Together, the isolation of augmented reality as a variable with the valuable comparative information on engagement levels will begin to reveal insights on the contribution of augmented reality to the visitor experience in a museum setting, and particularly within a Victorian-aged exhibit. Visitors will be inquired about the importance of the visual dimension of the exhibit to their overall experience and the cognitive constructions that may be attributed to those visual interpretations. Future publications will report on the findings of the study.

The collected knowledge provided by this research is in support of the advantages that a stronger interest by museum professionals for visual literacy in natural history museums can bring to the visitor experience. It also emphasizes the positive role augmented reality visualizations can play in developing higher engagement levels at museums and in connecting visitors, objects and their unique stories.

\section{ACKNOWLEDGMENTS}

This research is supported by the co-funding of the European project POPH/FSE and a grant from the Foundation for Science and Technology (SFRH/BD/51840/2012), under the UT Austin| Portugal, CoLab.

\section{REFERENCES}

Azuma, R. (1997) A Survey of Augmented Reality. Presence: teleoperators and virtual environments, 6(4), pp.355-385.

Bain, R. and Ellenbogen, K. (2002) Placing objects within disciplinary perspectives: examples from history and science. In Paris, S. (ed), Perspectives on Object-Centered Learning in Museums, Lawrence Erlbaum Associates, Mahwah, NJ.

Borun, M. and Miller, M. (1980) What's in a Name? A Study of the Effectiveness of Explanatory Labels in a Science Museum. Franklin Institute Science Museum and Planetarium, Philadelphia, PA.

Falk, J. and Dierking, L. (2000) Learning from museums: visitor experiences and the making of meaning. Altamira Press, Lanham, MD.

Gilbert, J. (2005) Visualization in Science Education. Springer, Dordrecht.

Johnson, L. et al. (2012) The NMC Horizon Report: 2012 Museum Edition. The New Media Consortium, Austin, TX.

Jones, J. (2002) The art and science of seeing: applying visual literacy interpretation in natural history museums. Curator: The Museum Journal, 45(4), pp.257-261.

Kahr-Højland, A. (2006) Changing paradigms within museum exhibitions: the use of mobile technologies within semi-formal learning settings. Network of Design \& Digital Heritage (NODEM 06). Oslo, 7-9 December 2006, 1-14.

McManus, P. (1989) Oh, yes, they do: how museum visitors read labels and interact with exhibit texts. Curator: The Museum Journal, 32(3), pp.174-189.

Parry, R. (2012) The End of the Beginning: museums and the naturalizations of digital media. The Transformative Museum, Roskilde, 23-25 May 2012.

http://www.dreamconference.dk/?page_id=1385 (retrieved 1 April 2013).

Perkins, D. (1994) The Intelligent Eye: Learning to Think by Looking at Art. J. Paul Getty Trust, Los Angeles, CA.

Proctor, N. (2012) Introduction: what is mobile? In Proctor N. (ed), Mobile Apps for Museums: the $A A M$ guide to planning and strategy. The AAM Press, Washington, DC.

Reiss, M. and Tunnicliffe, S. (2011) Dioramas as depictions of reality and opportunities for learning in biology. Curator: The Museum Journal, 54(4), pp.447-459. 
Rice, D. (1988) Vision and culture: the role of museums in visual literacy. The Journal of Museum Education, pp.13-17.

Schavemaker, M. (2012) Is augmented reality the ultimate museum app? Some strategic considerations. In Proctor, N. (ed), Mobile Apps for Museums: the AAM guide to planning and strategy. The AAM Press, Washington, DC.

Snyder, S. and Elinich, K. (2010) Augmented reality for interpretive and experiential learning. Electronic Visualisation and the Arts (EVA 2010), London, UK, 5-7 July 2010, 87-92. British Computer Society, London.

Tallon, L. (2013) Museums \& Mobile Survey 2013. Pocket-Proof \& LearningTimes. http://www.museums-mobile.org/survey/ (retrieved 1 April 2013).

Tunnicliffe, S. (1998) Skeletons-putting the flesh on the bones. The Informal Science Review, 32(1), pp.8-11.

Tunnicliffe, S. and Scott, A. (2003) Observations made by primary school children at skeleton exhibits in a natural history museum. Journal of Education in Museums, 24, pp.40-44.

Weng, E. et al. (2011) Augmented Reality for Museum Artifacts Visualization. International Journal of Computer Science and Information Security, 9(5), pp.174-185. 